\title{
Estimativa da evapotranspiração real utilizando geotecnologias na região do semiárido, Bahia, Brasil
}

A evapotranspiração é um importante componente do ciclo hidrológico e suas informações quantitativas são essenciais para o manejo sustentável dos recursos hídricos. Diante disso, o objetivo deste estudo foi estimar a Evapotranspiração Real (ETr) diária da Bacia Hidrográfica do Rio Jacaré (BHRJ), localizado na microrregião de Irecê no Estado da Bahia. A metodologia do estudo foi baseada na utilização de ferramentas de geoprocessamento em conjunto com o algoritmo SEBAL para se estimar os componentes do balanço de energia no período de 30/01/2019 à 27/09/2019. Dentre os resultados obtidos, verificou-se que os valores de EA e ER para o dia 30 de janeiro de 2019 foi de $4,76 \mathrm{~mm}$ dia-1 e 48, 3\%, respectivamente. Além disso, os menores erros encontrados foram para o dia $27 / 09 / 2019$ obtendo EA de 0,08 mm dia-1 e ER de $3 \%$. Por fim, espera-se que os resultados alcançados por meio deste estudo sirvam de base para a gestão sustentável dos recursos hídricos das bacias hidrográficas, principalmente as inseridas na região do Nordeste brasileiro.

Palavras-chave: Algoritmo SEBAl; Recursos hídricos; Balanço de energia; Meio ambiente; LANDSAT 8.

\section{Estimate of real evapotranspiration using geotechnologies in the semiarid region, Bahia, Brazil}

\begin{abstract}
Evapotranspiration is an important component of the hydrological cycle and its quantitative information is essential for the sustainable management of wate resources. Therefore, the objective of this study was to estimate the daily Real Evapotranspiration (ETr) of the Jacaré River Basin (BHRJ), located in the micro-region of Irecê in the State of Bahia. The methodology of the study was based on the use of geoprocessing tools in conjunction with the SEBAL algorithm to estimate the components of the energy balance in the period from 01/30/2019 to 09/27/2019. Among the results obtained, it was found that the AE and ER values for January 30,2019 were $4.76 \mathrm{~mm}$ day- 1 and $48,3 \%$, respectively. In addition, the smallest errors found were for 09/27/2019 obtaining EA of $0.08 \mathrm{~mm}$ day-1 and ER of $3 \%$. Finally, it is expected that the results achieved through this study will serve as a basis for the sustainable management of water resources in hydrographic basins, especially those inserted in the Northeast region of Brazil.
\end{abstract}

Keywords: SEBAL algorithm; Water resources; Energy balance; Evirionment; LANDSAT 8.

Topic: Engenharia Ambiental

Reviewed anonymously in the process of blind peer.
Received: 03/06/2021

Approved: 27/06/2021
José Ludemario da Silva Medeiros (D) Universidade Estadual da Paraíba, Brasil http://lattes.cnpq.br/4084311510300833 http://orcid.org/0000-0002-7523-9601 joseludemariomedeiros@gmail.com

luri Souza de Jesus (iD)

Universidade Federal da Campina Grande, Brasil

http://lattes.cnpq.br/9940532745543327 http://orcid.org/0000-0003-0902-2997 moixaiuri@gmail.com

Tássio Jordan Rodrigues Dantas da Silva (it) Universidade Estadual da Paraíba, Brasil http://lattes.cnpq.br/1991160864996557 http://orcid.org/0000-0002-1708-5472 tassiojordan@hotmail.com
Maxsuel Bezerra do Nascimento (iD) Universidade Estadual da Paraíba, Brasil http://lattes.cnpq.br/7100621157794567 http://orcid.org/0000-0001-6381-2688 maxsuel10gba@hotmail.com

\section{Janaína Aparecida Cezário (iD}

Universidade Estadual da Paraíba, Brasil http://lattes.cnpq.br/2080075497375535 http://orcid.org/0000-0002-9866-4168 ja_nayna2@yahoo.com.br

William de Paiva (D)

Universidade Estadual da Paraíba, Brasil http://lattes.cnpq.br/2612977983185686 http://lattes.cnpq.br/261297798318568 w.paiva461@gmail.com
Laércio Leal dos Santos (iD)

Universidade Estadual da Paraíba, Brasil http://lattes.cnpq.br/6344196123061264 http://orcid.org/0000-0002-8175-4378 laercioeng@yahoo.com.br

\section{Gabriella Moreira Campos (iD}

Universidade Federal Rural de Pernambuco, Brasil

http://lattes.cnpq.br/5534366996875730

http://orcid.org/0000-0002-7227-0750 moreiragabriella84@gmail.com

\section{Elicarla Barbosa Moitinho}

Universidade Federal Rural de Pernambuco, Brasil

http://lattes.cnpq.br/5793492935537870

elicarlamoitinho@gmail.com
Lucas Leite da Silva Fonseca Universidade do Estado da Bahia, Brasil http://lattes.cnpq.br/6472938525334292 ucas29r@hotmail.com d

DOI: $10.6008 / C B P C 2179-6858.2021 .006 .0030$
Referencing this:

MEDEIROS, J. L. S.; JESUS, I. S.; SILVA, T. J. R. D.; NASCIMENTO, M. B.; CEZARIO, J. A.; PAIVA, W.; SANTOS, L. L.; CAMPOS, G. M.; MOITINHO, E. B.; FONSECA, L. L. S.. Estimativa da evapotranspiração real utilizando geotecnologias na região do semiárido, Bahia, Brasil. Revista Ibero Americana de Ciências Ambientais, v.12, n.6, p.360376, 2021. DOI: http://doi.org/10.6008/CBPC2179$\underline{6858.2021 .006 .0030}$ 


\section{INTRODUÇÃO}

A Evapotranspiração (ET) é um processo combinado de perda de água na atmosfera pela evaporação do solo e transpiração da vegetação (ALLEN et al., 2002). O conhecimento da ET das culturas e da vegetação é uma importante ferramenta na gestão de bacias hidrográficas e modelagens meteorológicas (BEZERRA, 2008), e dentre as parcelas que compõe o ciclo hidrológico, a ET é uma das que apresentam maior incerteza pela dificuldade de ser medida e requerer métodos indiretos para a sua estimativa, onde as metodologias mais utilizadas precisam de dados meteorológicos restritos a um local. Além disso, a ET sofre influência no espaço e tempo nos parâmetros das propriedades do solo, uso do solo, vegetação, disponibilidade de água e relevo (GIACOMONI, 2005).

Vários métodos indiretos para estimativas de ET são apresentados na literatura, destacando-se: Método de Thornthwaite, Penman-Monteith, Tanque Classe A (TCA), Método da Radiação Solar, dentre outros. Cabe ressaltar que todas essas metodologias falham quando o objetivo é a obtenção da ET em extensas áreas com heterogeneidade. Diante disso, uma alternativa para sanar esse problema é a utilização de imagens de satélites, pois apresentam várias vantagens, como por exemplo: Alta qualidade nos resultados, agilidade e as imagens cobrem regiões distintas com características diversas.

Dentre os modelos que utilizam imagens de satélites e técnicas de sensoriamento remoto destacase o Surface Energy Balance Algorithm for Land (SEBAL), proposto por Bastiaanssen et al. (1998). O SEBAL é um algoritmo que utiliza imagens de satélite e poucos dados de superfície para estimar as componentes do balanço de energia, e tem sido utilizado amplamente pela comunidade científica, na maioria dos países, para estimar diversos parâmetros da superfície terrestre para diferentes regiões geográficas.

O algoritmo SEBAL apresenta diversas vantagens, pois requer dados mínimos de campos, e imagens de satélites de qualquer sensor orbital que efetue medidas de radiação eletromagnética na região do visível, infravermelho próximo e infravermelho termal como o LANDSAT-5 (TM), utilizados por: Menezes (2011); Bezerra (2008); Machado (2014); Silva et al. (2006) e o LANDSAT-8 (OLI) utilizados por: Silva et al. (2018). Além disso, o SEBAL refere-se a um método viável economicamente de estimativa dos componentes do balanço de energia, já que as medições dos componentes energéticos são feitas por sensores específicos e de altos custos.

Diante deste contexto, cabe ressaltar que o conhecimento da água perdida por ET é fundamental para o conhecimento do balanço hídrico de uma determinada região. Além disso, a ET influência diretamente os rendimentos das bacias hidrográficas, a umidade atmosférica, a determinação da capacidade de reservatórios, regime de chuvas, dentre outros. Diante disso, o algoritmo SEBAL surgi como uma importante ferramenta para estimativas de ET, principalmente em áreas com baixa pluviosidade, como é o caso da Bacia Hidrográfica do Rio Jacaré (BHRJ), localizada na Microrregião de Irecê - BA.

A Microrregião de Irecê está inserida na região central do Estado da Bahia, no domínio das bacias hidrográficas dos rios Verde e Jacaré, afluentes da margem direita do Rio São Francisco. Segundo Ramos (2016) essa região apresentou entre os anos de 1980 a 1990 um grande potencial nas atividades ligadas ao 
setor econômico de cunho agrícola, destacando-se na produção de feijão, milho e mamona. Entretanto, após a década de 90 do século XX, ocorreu o declínio da produção destes grãos ocasionada por contas das perdas de safras causadas pelas secas (Fenômeno natural que causa longos períodos de estiagens, ocorrendo com maiores frequências nas regiões ariadas e semiáridas) e a redução dos serviços governamentais de apoio (crédito e financiamento), onde houve uma diversificação das atividades agropecuárias, através das culturas irrigadas e pecuária de gado.

Neste contexto, cabe frisar que as atividades agropecuárias apresentam grande potencial impactante para o meio ambiente, destacando-se a pecuária bovina no modelo extensivo. Em síntese, essa atividade promove a destruição da vegetação natural de extensas áreas, principalmente das matas ciliares, assim, consequentemente, reduzindo a quantidade e prejudicando a qualidade dos recursos hídricos.

Nesse cenário que a temática ambiental surge em virtude dos desmatamentos e comprometimentos dos recursos hídricos ligadas as atividades agropecuárias no modelo convencional, a estimativa da ET utilizando o algoritmo SEBAL compreende uma importantíssima ferramenta na geração de subsídios para o planejamento e gestão dos recursos hídricos.

Diante do contexto apresentado, o objetivo deste estudo foi estimar a Evapotranspiração Real (ETr) diária na BHRJ, localizado no Estado da Bahia, a partir do algoritmo SEBAL.

\section{MATERIAIS E MÉTODOS}

\section{Localização da Área de Estudo}

A área de estudo encontra-se situada na parte central do estado da Bahia, na BHRJ (Figura 1), com uma área e comprimento de drenagem de aproximadamente $393,295 \mathrm{~km}^{2}$ e 465,577 km, respectivamente. A BHRJ abrange os municípios de Lapão (0,225 \%), Presidente Dutra (4,0388 \%), Irecê (74,84 \%), João Dourado $(17,715$ \%) e São Gabriel (3,171 \%).

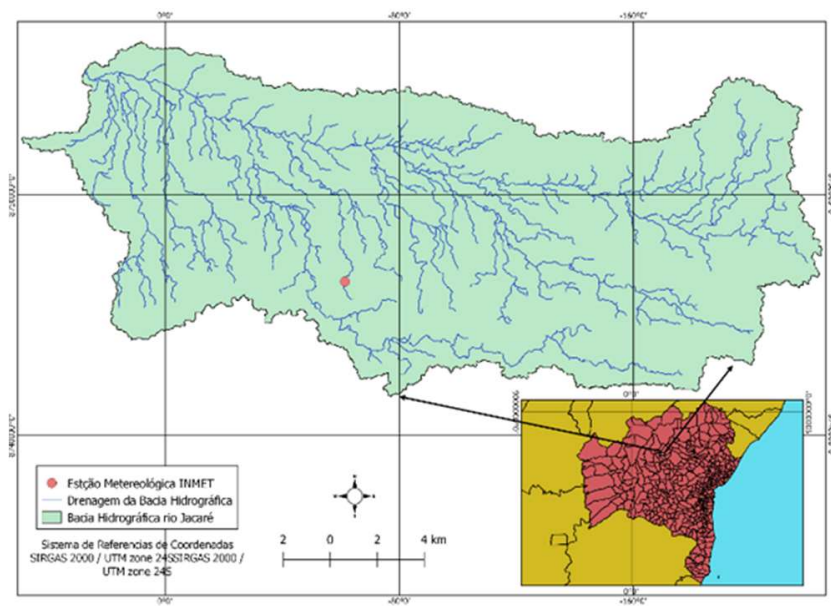

Figura 1: Mapa de localização da área de estudo.

\section{Caracterização da Área de Estudo}

A área do estudo apresenta clima BSwh (semiárido, tipo estepe, muito quente, com estação chuvosa 
no verão que se atrasa para o outono, podendo não ocorrer) segundo a classificação climática de Köppen. Além disso, a precipitação média anual é de $600 \mathrm{~mm}$, apresentando pluviosidade irregular entre o final do mês de setembro e o final do mês de abril do ano seguinte. Segundo Gonçalves (2018) a ET anual é de aproximadamente $1300 \mathrm{~mm}$, assim, enquadrando a região no polígono das secas.

\section{Delimitação da BHRJ}

A delimitação da BHRJ foi por meio do software Qgis, versão 2.14.1, utilizando o algoritmo r.watershed (Programa de Criação de Bacias Hidrográficas). Para tal, inicialmente foi necessário a obtenção do Modelo Digital de Elevação (MDE), com resolução espacial de $30 \mathrm{~m}$, obtido no site do serviço geológico dos Estados unidos da América do Norte, United States Geological Survey (USGS).

\section{Tratamento das Imagens e Calibração do Algoritmo SEBAL}

Para a estimativa de ETr usando algoritmo SEBAL utilizou-se das imagens do LANDSAT 8 obtidas de forma gratuita através do site do USGS. Para tanto, inicialmente foram estabelecidos dois critérios para a seleção das imagens. Deste modo, o primeiro foi referente a porcentagem de nuvens, onde foram selecionadas as cenas que possuíssem a menor interferência das nuvens para se obter uma boa visualização da área do estudo. Por fim, o segundo critério para as escolhas das cenas foram as datas, correspondendo sempre a uma imagem durante o período chuvoso e a outra durante a estiagem.

Após a obtenção das imagens, as mesmas foram configuradas para o mesmo sistema de referência no DATUM horizontal SIRGAS 2000 nas coordenadas Universal Transversa de Mercator (UTM) na zona 24 Sul. Logo após, realizou-se o recorte das imagens por meio de uma camada máscara com a delimitação da área de estudo, com o auxílio do plug-in Semi-Automatic Classification Plugin (SCP) no software Qgis, versão 2.14.11.

A estimativa da ETr foi através do algoritmo SEBAL em conjunto com o software Qgis, versão 7.0.0. Para tal, inicialmente foi inserido no ambiente do Qgis o recorte das imagens reprojetadas, como também o arquivo do algoritmo e os metadados correspondentes para cada data das cenas escolhidas. Nesta mesma interação, foi inserida a cena do sensor Advanced Spaceborn Thermal Emission and Reflection Radiometer (ASTER), capaz de estimar os componentes relacionados às trocas de radiação e energia entre a atmosfera e a superfície através do senso radiométrico. Cabe ressaltar, que este procedimento foi realizado para uma cena por vez.

Para a calibração do algoritmo recorreu-se primeiramente da velocidade do vento, em seguida da Evapotranspiração de Referência no Horário $\left(\mathrm{ET}_{\mathrm{i}}\right)$ da passagem do satélite e por último da Evapotranspiração de Referência Diária (ETO) no dia da passagem do satélite. Para obtenção dos cálculos ETO e ET i utilizou-se da Equação do método de Penman-Monteith (Equação 1). Além disso, os dados para obtenção da ETr e velocidade do vento foram obtidos no Instituto Nacional de Meteorologia (INMET) e na estação meteorológica de Irecê. 
$\operatorname{ET0}=\frac{0,408 \Delta\left(R_{n}-G\right) \frac{\gamma 900 U_{2}\left(e_{s}-e_{a}\right)}{T+273}}{\Delta+\gamma\left(1+0,34 U_{2}\right)}$

(Equação 1)

Para os valores $\mathrm{ET}_{\mathrm{i}}$ foi aplicada a média horária a partir do valor do ETO, uma vez que os dados obtidos no INMET para a determinação de ETO são diários e não condiz com os horários da passagem dos satélites. Os dados utilizados para calibração do SEBAL bem como os horários e datas das passagens dos satélites estão expostos na Tabela 1.

Tabela 1: Dados utilizados para a calibração do SEBAL.

\begin{tabular}{lllll}
\hline Satélite & Data & Velocidade do vento $(\mathbf{m} / \mathbf{s})$ & ET $_{\mathbf{i}}$ & ETO \\
\hline LANDSAT 8 (OLI) & $30 / 01 / 2019$ & 3,33 & 0,22 & 5,15 \\
LANDSAT 8 (OLI) & $27 / 09 / 2019$ & 2,43 & 0,12 & 2,75 \\
\hline
\end{tabular}

\section{Algoritmo SEBAL para Determinação da Evapotranspiração Real}

Para a determinação da ETr, inicialmente o algoritmo SEBAL necessitou das informações do balanço completo de radiação de energia incidente sobre a superfície terrestre convertendo ( $\left.\mathrm{W} \mathrm{m}^{-2}\right)$ para $(\mathrm{mm})$.

Onde o Fluxo de Calor Latente (LE) é obtido a partir da diferença entre Saldo de Radiação (Rn), Densidade de fluxo de calor sensível $\left(\mathrm{W} \mathrm{m}^{-2}\right)$ e Densidade de Fluxo de Calor Sensível $(\mathrm{H})$ e a Densidade de Fluxo de Calor no Solo (G), conforme a Equação 2:

$$
\mathrm{LE}=\mathrm{Rn}-\mathrm{G}-\mathrm{H} \quad \text { (Equação 2) }
$$

\section{Saldo de Radiação Instantâneo (Rn)}

O Rn foi determinado pela Equação 3, baseada na metodologia de (BASTIAANSSEN, 1995; MORSE et al., 2001; ALLEN et al., 2002; SILVA et al., 2006):

$$
\mathrm{Rn}=(1-\alpha) \text { Rsi }+ \text { RLo }- \text { RLi }-\left(1-\varepsilon_{0}\right) \text { RLo (Equação 3) }
$$

Em que Rsi é a radiação de onda curta incidente $\left(\mathrm{W} \mathrm{m}^{-2}\right)$, RLo é a radiação de onda longa emitida ( $\left.\mathrm{W} \mathrm{m}^{-2}\right)$, RLi é a radiação de ondas curtas incidentes $\left(\mathrm{W} \mathrm{m}^{-2}\right), \alpha$ é o albedo de superfície (adimensional) e $\varepsilon_{0}$ é a emissividade de superfície também adimensional.

\section{Fluxo de Calor no Solo (G)}

O fluxo de calor no solo foi obtido através do modelo empírico desenvolvido por Bastiaanssen (2000) para valores próximos ao meio dia.

$$
G=\left[\begin{array}{c}
\left(\frac{T s-273,15}{\alpha}\right) \times \\
\left(0,0038 \alpha+0,0074 \alpha^{2}\right)\left(1-0,98 N_{D V I}^{4}\right)
\end{array}\right] \text { Rn (Equação 4) }
$$

Em que Ts (으) é a temperatura de superfície; e NDVI é Índice de Vegetação da Diferença Normalizada calculado em cada pixel através da equação abaixo:

$$
\text { NDVI }=\frac{\rho_{2}-\rho_{1}}{\rho 2+\rho_{1}} \quad \text { (Equação 5) }
$$

Sendo $\rho_{1}$ e $\rho_{2}$ as bandas espectrais (b4) vermelho e (b5) infravermelho próximo do LANDSAT-8. Para NDVI <0, considerou G =0,3Rn como também fizeram Silva et al. (2006) e Coelho (2016). 


\section{Temperatura de Superfície (Ts)}

A partir das bandas termais (B6) infravermelho termal e (B10) do LANDSAT 8, foi possível calcular a Temperatura de superfície (Ts) pela Equação 6, onde $K_{1}$ (K) são as bandas termais e Enb a Emissividade.

$$
\text { Ts }=\frac{\left(\mathrm{K}_{1}\right)}{\left(\frac{1+\ln \left(10.8 \mathrm{~K}_{1}\right)}{14380}\right)(\ln (\text { Enb }))} \text { (Equação 6) }
$$

\section{Fluxo de Calor Sensível (H)}

Para determinar o H no algoritmo SEBAL utilizou-se a Equação 7, conforme utilizada por Silva et al. (2018).

$$
\mathrm{H}=\frac{\rho_{\mathrm{a}} \mathrm{c}_{\mathrm{p}(\mathrm{dt})}}{\mathrm{R}_{\mathrm{ah}}} \text { (Equação 7) }
$$

Sendo $\rho_{\mathrm{a}}$ a densidade do ar úmido $\left(1,5 \mathrm{~kg} \mathrm{~m}^{-3}\right) ; C_{\mathrm{p}}$ é o calor especifico do ar $\left(1004 \mathrm{JK}^{-1} \mathrm{~K}^{-1}\right)$; dt é a diferença de temperatura $\left(T_{s 1}-T_{S 2}\right)$ e do $\operatorname{ar}\left(Z_{1}\right.$ e $\left.Z_{2}\right)$ e $R_{a h}$ é a resistência aerodinâmica ao transporte de calor sensível (s/m).

A resistência aerodinâmica é calculada para obter uma instabilidade neutra a partir da rugosidade de superfície, deslocamento de altura e instabilidade térmica da atmosfera (OLIVEIRA, 2012; COELHO, 2016), conforme a Equação 8.

$$
\mathrm{R}_{\mathrm{ah}}=\frac{\ln \left(\frac{\mathrm{Z}_{2}}{\mathrm{Z}_{1}}\right)}{\mathrm{U}_{\mathrm{map}} \mathrm{K}} \quad \text { (Equação 8) }
$$

$\mathrm{Na}$ qual $Z_{1}$ e $Z_{2}(m)$ são as alturas acima do dossel vegetativo sendo 0,1 e 2 , respectivamente; $K$ a constante de Von Karman $(0,41)$; e $\cup_{m a p}$ é a velocidade de fricção inicial calculada em cada pixel. Para a velocidade de friç̧ão na estação meteorológica $\left(U_{\text {ast }}\right)$ considerou a equação do perfil logaritmo do vento em condições de instabilidade neutra (OLIVEIRA, 2012; COELHO, 2016; SILVA et al., 2006), por meio da Equação 9:

$$
\mathrm{U}_{\mathrm{ast}}=\frac{\mathrm{ku}_{\mathrm{x}}}{\ln \left(\frac{\mathrm{z}_{\mathrm{x}}}{\text { zom }_{\text {estação }}}\right)} \text { (Equação 9) }
$$

Sendo $k$ é a constante de von Karman $(0,41) ; u_{x}$ a velocidade do vento $\left(\mathrm{m} / \mathrm{s}^{2}\right)$ na altura $Z_{x}$ que corresponde a altura do sensor instalado na estação meteorológica experimental no local da pesquisa. Neste trabalho utilizou-se $Z_{x}=2 \mathrm{~m}$; zom estação o coeficiente de rugosidade do local em função da altura média da vegetação, onde, onde $\mathrm{h}$ é a altura média da vegetação $(\mathrm{m})$ na área de medição da velocidade do vento considerado neste trabalho $h=0,15$ (BRUTSAERT, 1982), conforme a Equação 10.

$$
\text { zom }_{\text {estação }}=0,123 h \text { (Equação 10) }
$$

A velocidade do vento a uma altura de 200 m (U (U200_estação) foi projetada admitindo-se a atmosférica assumindo nenhum efeito de rigidez da superfície (blending height). Para isso, utilizou-se a Equação 11 proposta por (BASTIAANSSEN et al., 1998). 


$$
\mathrm{U}_{200 \_ \text {estação }}=\mathrm{U}_{\mathrm{ast}} \ln \frac{\left(\frac{200}{\text { zomestação }}\right)}{\mathrm{k}} \text { (Equação 11) }
$$

$O$ coeficiente de rugosidade local zom_estação $(m)$, velocidade de friç̧ão $U_{\text {ast }}\left(\mathrm{m} \mathrm{s}^{-1}\right)$ e velocidade do

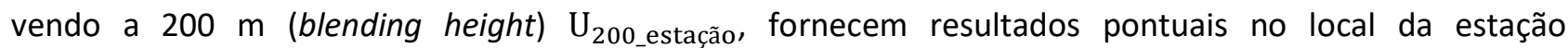
meteorológica experimental.

Para a determinação da velocidade de fricção inicial $U_{\text {nicial_ }}\left(\mathrm{m} \mathrm{s}^{-1}\right)$, pixel a pixel, utilizou-se a $U_{\text {ast }}$, assim admitindo a velocidade do vento $\left(u_{x}\right)$ na altura $(z)$ de 200 m, conforme a Equação 12.

$$
\mathrm{U}_{\text {inicial }}=\frac{\mathrm{ku}_{200}}{\ln \left(\frac{200}{\mathrm{z}_{0 \mathrm{~m}}}\right)} \text { (Equação 12) }
$$

Na qual $Z_{0 m}$ é o coeficiente de rugosidade obtido em função do SAVI a partir da Equação 13 utilizada por Bastiaanssen (2000).

$$
\mathrm{Z}_{0 \mathrm{~m}}=\exp (-5,809+5,62 \mathrm{SAVI}) \text { (Equação 13) }
$$

Para se obter o H é preciso definir a diferença de temperatura do $\operatorname{ar}\left(\mathrm{T}_{\mathrm{ar}}\right)$ e de superfície (Ts), definido como (dt) em kelvin, como uma relação linear, uma vez que, a temperatura do ar e $R_{a h}$ são desconhecidos. Logo, possibilita que o dt seja determinado por um processo de calibração, onde o SEBAL utiliza dois pixels âncoras: o "pixel frio" e o "pixel quente", definido neste trabalho como "pcold" e "phot" respectivamente. Por fim, o valor de dt é obtido pela Equação 14 de (BASTIAANSSEN et al., 1998).

$$
\mathrm{dt}=\mathrm{aTs}+\mathrm{b}
$$

Sendo a e b os coeficientes de correlação tomando como base os componentes do balanço de energia dos pixels âncoras; e Ts a temperatura de superfície dos pixels em questão. Além disso, também considerou para o Pcold o fluxo de calor sensível sendo nulo, admitindo-se então $\mathrm{H}_{\text {cold }}=0, \mathrm{e}$, portanto, para dt assumindose o valor de 0 . Para o $\mathrm{H}_{\text {hot }}$ o fluxo de calor latente é nulo $\left(\mathrm{W} \mathrm{m}^{-2}\right)$ e o fluxo de calor sensível foi determinado pela Equação 15 (OLIVEIRA, 2012; COELHO, 2016; BASTIAANSSEN, 2000).

$$
\mathrm{H}_{\text {hot }}=\mathrm{Rn}-\mathrm{G}=\left(\frac{\rho \mathrm{C}(\mathrm{a}+\mathrm{bTs})}{\mathrm{Rah}}\right) \text { (Equação 15) }
$$

Em que Ts, Rn, G e rah correspondem aos valores coletados nas cenas que foram processadas exatamente nas coordenadas (east; north) do pixel quente.

Para a geração dos mapas pcold e phot foi considerada as seguintes condições: para pcold (NDVI>0,4 utiliza-se Ts $<T_{\text {média }}$ e para Phot (SAVI $>0,18$ e SAVI $<0,3$, utiliza-se Ts).

A escolha dos pixels pcold e phot deu-se de forma semiautomática uma vez que a partir dos mapas gerados pelo SEBAL. Para tanto, utilizou-se das coordenas (east e north) dos mapas para o período do estudo, de 30 de janeiro à 27 de setembro do ano de 2019, utilizando como critério neste trabalho a aleatoriedade para escolha dos pixels. Logo, a partir das informações colhidas em cada pixel de "pcold" e "phot" pôde-se determinar os coeficientes de correlação (a e b), por meio das Equações 16 e 17.

$$
\begin{gathered}
\mathrm{a}=(\text { Rnphot }- \text { Gphot }) * \frac{\text { Rahphot }}{(\text { Tsphot-Tsco }) * \operatorname{Cp} * \rho} \text { (Equação 16) } \\
\mathrm{b}=\mathrm{a} * \text { Tspcold (Equação 17) }
\end{gathered}
$$

$\mathrm{Na}$ qual Rnphot, Gphot e Rahhot correspondem aos valores do saldo de radiação $\left(\mathrm{W} \mathrm{m}^{2}\right)$, fluxo de 
calor no solo $\left(\mathrm{W} \mathrm{m}^{-2}\right)$ e resistência aerodinâmica ao fluxo de calor sensível $\left(\mathrm{s} \mathrm{m}^{-1}\right)$ coletados no pixel quente; Tsphot e Tspcol são as temperaturas de superfície dos pixels "phot"e "pcold" respectivamente; Cp o calor especifico do ar (1004 $\left.\mathrm{JK}^{-1} \mathrm{~K}^{-1}\right)$; e $\rho$ a densidade dar, considerada para esse estudo como 1,25 $\left(\mathrm{kg} \mathrm{m}^{-3}\right)$.

A partir dos valores obtidos de "a" e "b" pôde-se determinar o $\mathrm{H}$ para todos os pixels da cena. No entanto, os valores de $\mathrm{H}$ que foram obtidos não representam adequadamente o fluxo de calor sensível porque foram obtidos em condições de instabilidade neutra. Assim, foi preciso obter o comprimento de Monin-Obukhov (L) computado em função dos fluxos de calor e momentum e que possibilita a condição de instabilidade atmosférica para cada pixel no instante de passagem do Satélite.

$$
\mathrm{L}=-\frac{\rho \mathrm{CPU}_{\text {inicial }} \mathrm{TS}}{\mathrm{kgH}} \text { (Equação 17) }
$$

Na qual g é o módulo gravitacional terrestre $\left(9,82 \mathrm{~m} / \mathrm{s}^{-2}\right)$. Para o cálculo do $\mathrm{L}$, considerou-se a condição de neutralidade e para os valores de $L$ definindo-se as condições de estabilidade da seguinte forma: se $L<0$, a atmosfera é considerada instável; se $L>0$, atmosfera é considerada estável; e se $L=0$, a atmosfera é considerada neutra. Para as correções de estabilidade da atmosfera e para o transporte momentum $\left(\psi_{\mathrm{m}}\right)$ e de calor $\left(\psi_{h}\right)$ utilizou-se as formulações de Paulson (1970) exposto na Figura 2.

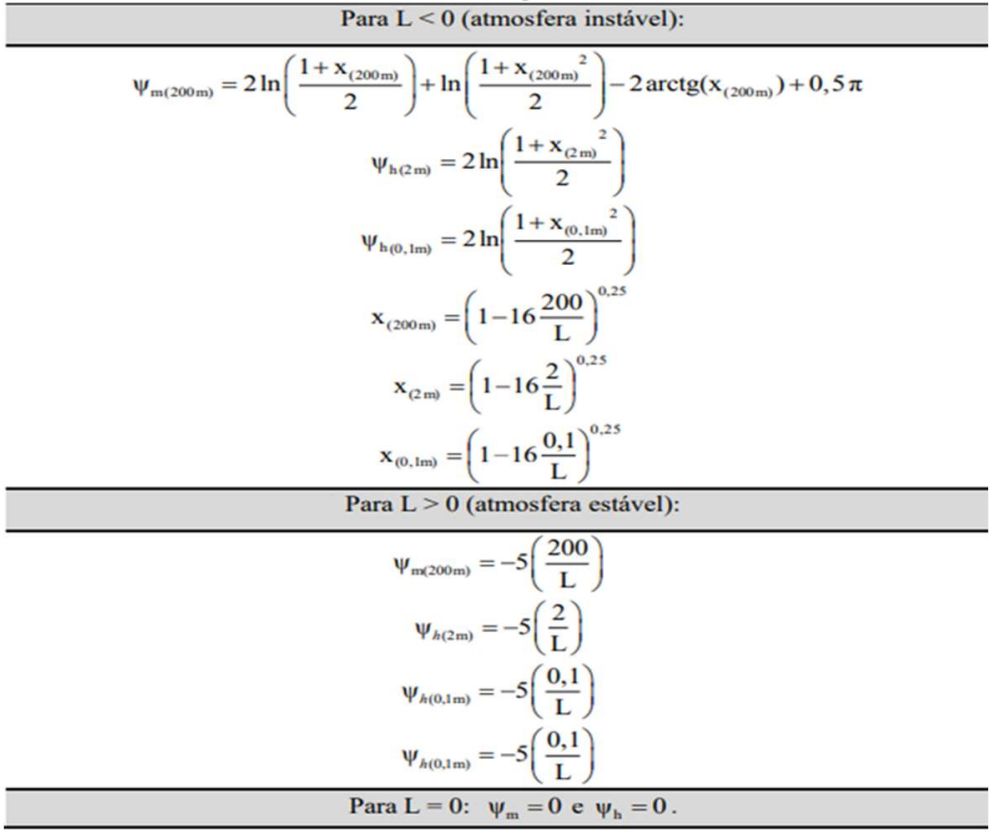

Figura 2: Equações para correção atmosférica $(\psi \mathrm{m})$ e $(\psi \mathrm{h})$. Fonte: Adaptado de Paulson (1970).

Sendo $\psi_{\mathrm{m}(200 \mathrm{~m})}$ a correção de estabilidade atmosférica para o transporte momentum na altura 200 $\mathrm{m} ; \psi_{\mathrm{h}(2 \mathrm{~m})}$ e $\psi_{\mathrm{h}(0,1 \mathrm{~m})}$ as correções de estabilidade atmosféricas para o transporte de calor nas alturas $2 \mathrm{~m}$ e 0,1 $m$, respectivamente; e $X(200 m), X(2 m)$ e $X(0,1 m)$ parâmetros. Considerando para a atmosfera neutra, tanto para $\left(\psi_{m}\right)$ quanto $\left(\psi_{h}\right)$ sendo iguais a 0 .

Após as correções atmosféricas foi possível corrigir a velocidade de fricção ( $\left.U_{\text {inicial_corrigido }}\right)$ e resistência

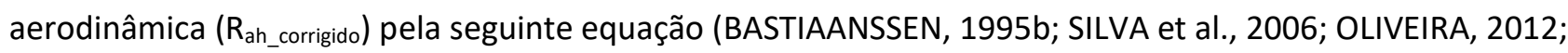
COELHO, 2016):

$$
U_{\text {inicial_corrigido }}=\frac{k_{u 200}}{\left.\ln \frac{200}{Z_{0 m}}\right)-\Psi_{m(200 m)}} \text { (Equação 18) }
$$




$$
R_{\text {ah_corrigido }}=\frac{\ln \left(\frac{Z_{1}}{z_{2}}\right)-\psi_{h(2 m)+} \psi_{h(0,1 m)}}{U_{\text {inicial_corrigido }} k}(\text { Equação 19) }
$$

$\mathrm{Na}$ qual $Z_{1}=0,1 \mathrm{~m}$ e $Z_{2}=2,0 \mathrm{~m}$ e $\Psi_{h(0,1 \mathrm{~m})}$ e $\psi_{h(2 m)}$ correspondem as correções de estabilidade para o transporte de calor sensível.

Calculado o H, G e Rn, pôde-se então obter o LET, que representa a taxa de calor latente da superfície devido a evapotranspiração. O LET, ETI e ETOF em $(\mathrm{mm})$ foram calculados pelas Equações 20, 21 e 22, respectivamente.

$$
\begin{gathered}
\mathrm{LET}=\mathrm{R}_{\mathrm{n}}-\mathrm{G}-\mathrm{H} \text { (Equação 20) } \\
\mathrm{ETI}=3600 *\left(\frac{\mathrm{LET}}{\lambda}\right)(\text { Equação 21) } \\
\mathrm{ET0F}=\frac{\text { ETI }}{\text { ETOi }} \text { (Equação 22) }
\end{gathered}
$$

Em que LET, Rn e G correspondem aos valores instantâneos do fluxo de calor latente $\left(\mathrm{W} \mathrm{m}^{-2}\right)$, saldo de radiação $\left(\mathrm{W} \mathrm{m}^{-2}\right)$ e fluxo de calor no solo $\left(\mathrm{W} \mathrm{m}^{-2}\right) ; \lambda$ equivale a $2,5 \times 10^{6} \mathrm{~J}$, energia necessária para ocorrer a evapotranspiração de 1,0kg de água; e ETOi é a evapotranspiração de referência no momento da passagem do satélite. Para o cálculo de Evapotranspiração real (ETr) utilizou-se a Equação 23.

$$
\mathrm{ETr}=\mathrm{ET0F} \times \mathrm{ET0} \text { (Equação 23) }
$$

Em que ETO é a evapotranspiração de referência obtida na estação meteorológica $(\mathrm{mm})$ calculado pela equação de Penman-Monteith.

\section{Análises Comparativas}

As análises comparativas foram realizadas entre os dados de evapotranspiração diária estimada pelo SEBAL de ETr e a ETO, onde foram calculadas pelo método de Penman-Monteith (Equação 24), parametrizado pela FAO (ALLEN et al., 1998). Este último método é considerado uma ET hipotética assumindo uma altura de vegetação de $0,12 \mathrm{~m}$, uma resistência de superfície fixa $70 \mathrm{sec} \mathrm{m}^{-1}$ e um albedo de 0,23 , assemelhando-se a ET de uma extensa superfície de grama verde e altura uniforme regada e com crescimento constante. Os dados para o cálculo de ETO foram obtidos no INMET na estação Irecê-A424.

$$
\text { ET0 }=\frac{0,408 \Delta\left(\mathrm{R}_{\mathrm{n}}-\mathrm{G}\right) \frac{\gamma 900 \mathrm{U}_{2}\left(\mathrm{e}_{\mathrm{s}}-\mathrm{e}_{\mathrm{a}}\right)}{\mathrm{T}+27}}{\Delta+\gamma(1+0,34 \quad 2)}(\text { Equação } 24)
$$

Em que ETO é a evapotranspiração de referência $\left(\mathrm{mm} \mathrm{dia}^{-1}\right) ; \Delta$ é a declividade da curva de pressão de vapor na saturação $\left(\mathrm{kPa}^{\circ} \mathrm{C}^{-1}\right)$; Rn é radiação líquida $\left(\mathrm{MJ} \mathrm{m}^{-2} \mathrm{dia}^{-1}\right)$; $\mathrm{G}$ é o fluxo de calor no solo ( $\mathrm{MJ}{ }^{\mathrm{m}-2} \mathrm{dia}^{-}$ ${ }^{1}$ ); $\gamma$ é a constante psicrométrica $\left(\mathrm{kPa}^{\circ} \mathrm{C}^{-1}\right)$; $\mathrm{T}$ é a temperatura média do $\mathrm{ar}$; $\mathrm{U}_{2}$ é velocidade do vento calculada para 2 metros de altura $(\mathrm{m} / \mathrm{s})$; $\mathrm{e}_{\mathrm{s}}$ é a pressão de saturação do valor d'água média diária (kPa); e e é a pressão parcial do vapor média diária $(\mathrm{KPa})$.

Os dados de ET estimados pelo SEBAL, corresponde à média aritmética do mapa de ETr, onde cada pixel desse mapa possui uma resolução espacial de $30 \mathrm{~m}$. Cabe ressaltar, que mais detalhes sobre o cálculo de ETO podem ser obtidos no Roteiro de Cálculo da Evapotranspiração de Referencia pelo Método de Penman-Monteith.

Para a análise estatística comparativa entre ETr e ETO foram utilizados os seguintes parâmetros estatísticos: utilizou-se o Erro Absoluto - EA ( $\mathrm{mm} \mathrm{dia}^{-1}$ ) e Erro Relativo - ER (\%). 


$$
\begin{gathered}
\mathrm{EA}=\left|\mathrm{X}_{\mathrm{ETr}}-\mathrm{X}_{\mathrm{ET0}}\right| \text { (Equação 25) } \\
\mathrm{ER}=\frac{\mathrm{X}_{\mathrm{ETr}}-\mathrm{X}_{\mathrm{ET0}}}{\mathrm{X}_{\mathrm{ETO}}}(\text { Equação 26) }
\end{gathered}
$$

Onde $\mathrm{XETr}_{\mathrm{E}}$ é o valor da evapotranspiração estimada pelo SEBAL; $\mathrm{X}_{\mathrm{ETO}}$ o valor da evapotranspiração calculada pelo método de Penman-Monteith.

\section{RESULTADOS E DISCUSSÃO}

\section{Parâmetros Físicos (NDVI, Temperatura de Superfície, Albedo de Superfície $(\alpha)$}

Durante o computo do algoritmo SEBAL são gerados três índices de vegetação: Índice da Diferença Normalizada (NDVI), Índice de Área Foliar (IAF) e Índice de Vegetação Ajustado aos Efeitos do Solo (SAVI).

Cabe ressaltar que o objetivo do NDVI é destacar a vegetação facilitando a compreensão dos índices vegetativos e solo exposto. Diante disso, na figura 3 estão apresentadas as variações do NDVI para a área do estudo.

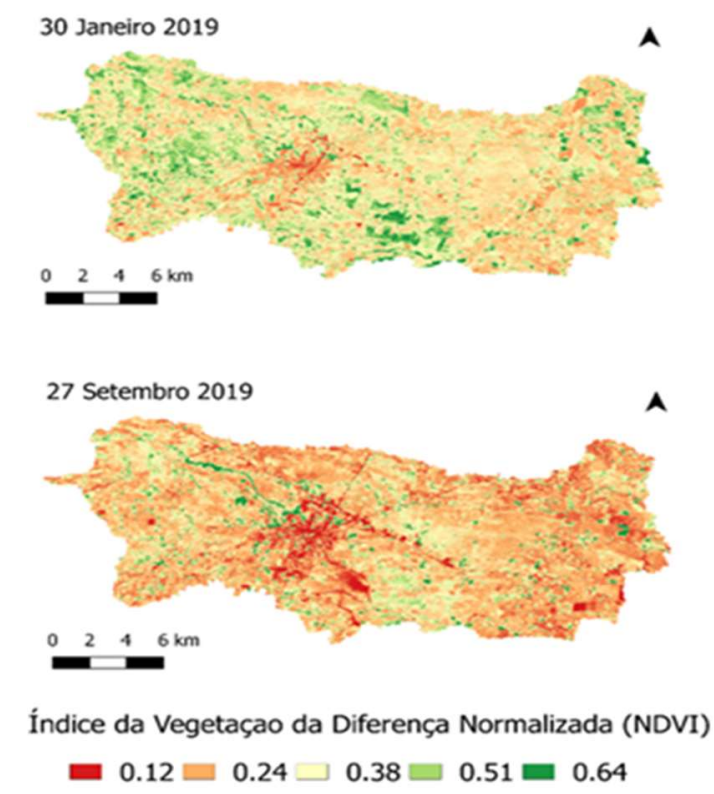

Figura 3: Mapa de NDVI para os meses de janeiro e setembro.

Por meio da Figura 3, verifica-se que as variações do NDVI para a data 30/01/2019 à 27/09/2019 foram as mesmas $(0,12-0,6)$, no entanto, dentro dessa faixa de variação a primeira data corresponde a um dossel vegetativo mais denso e vigorado devido ao mês estar inserido no período chuvoso da região.

O mês de setembro apesar de ter umidade e precipitação baixa, apresentam alguns pixels vigorados possuindo os valores próximos ao 0,64 , onde estes valores devem-se ao fato de os pixels corresponderem às áreas localizadas próximas aos cursos d'água e/ou áreas irrigadas. Outro fator que influencia na variação do NDVI para os meses de janeiro e setembro é o tipo de vegetação. Além disso, na região o bioma predominante é a caatinga, caracterizado pelas perdas de densidade de folhas durante os períodos de baixa pluviométrica e respostas rápidas da vegetação durante os períodos chuvosos.

Os valores mais baixos $(0,12-0,24)$ podem condizer com áreas degradadas, apresentando vegetação rala e solo exposto. Diante disso, cabe ressaltar que esses valores compreendem há extensas áreas da bacia 
hidrográfica relacionadas as atividades de cunho agropecuário, no período do estudo, sendo as mesmas bastante desenvolvida na bacia hidrográfica da área em estudo.

A Ts é utilizada pelo SEBAL para a determinação do $H, R n$ e $G$, além de ser requisito na obtenção dos pixels quente e frio. Já em relação ao NDVI e Ts, os mesmos comportam-se de maneira inversa, conforme exposto na Figura 4.

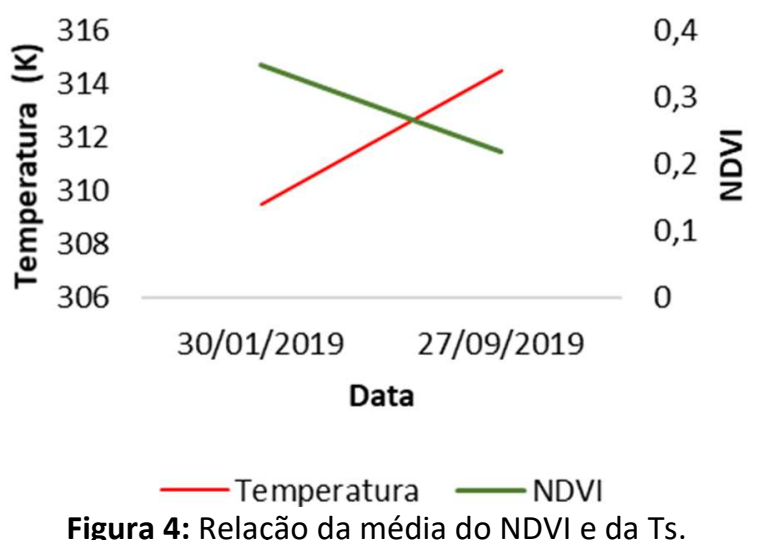

Com base na Figura 4, o observasse que as áreas com maior densidade vegetativa apresentaram temperaturas mais baixas. Por outro lado, locais que realizaram desatamento verificou-se temperaturas mais elevada. Na Figura 5 estão apresentadas as informações de Ts em graus Kelvin.

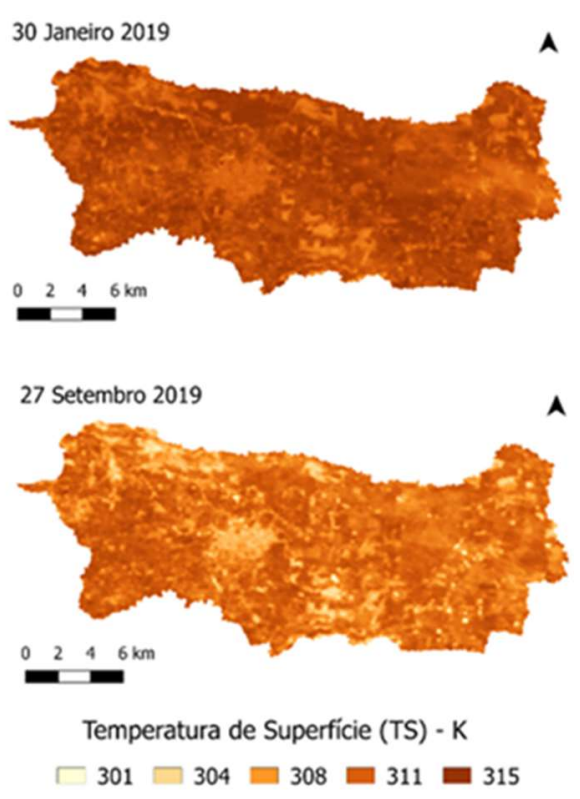

Figura 5: Mapas de Ts em graus Kelvin.

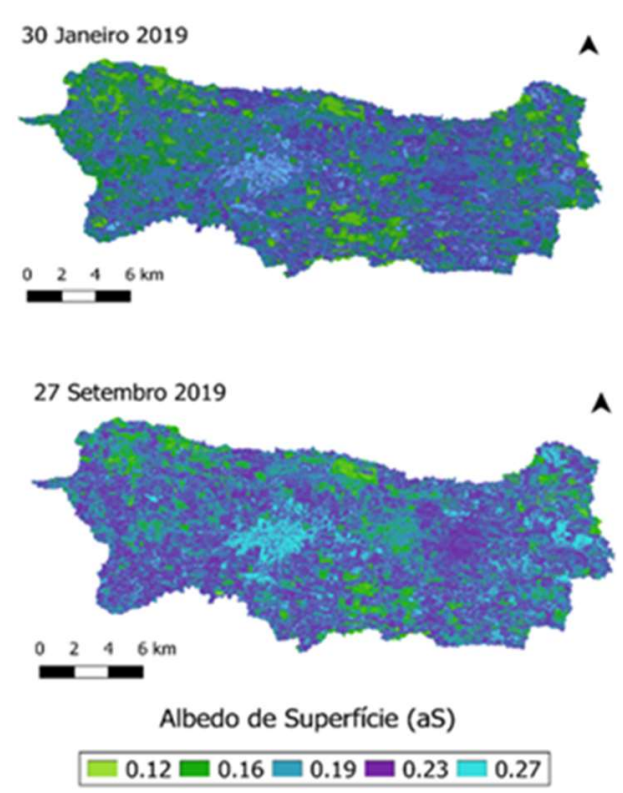

Figura 6: Mapas de albedo de superfície $(\alpha)$.

Com base na Figura 5, a primeira data está inserida no período chuvoso, onde a Ts média registrada foi de 214,5 (K) isso porque para esta data o NDVI teve média de 0,22 , fator determinante para a queda de temperatura na superfície, uma vez que as copas das árvores e mesmo a vegetação rala interceptam a radiação solar incidente e consequentemente impendem o aquecimento direto da superfície.

O albedo é o coeficiente de reflexão da radiação de onda curta incidente. Diante disso, na Figura 6 está ilustrada os valores de albedo variando 0,12 a 0,27 da área de estudo. 


\section{Componentes do Balanço de Energia (RN, G, H, LET).}

\section{Saldo de Radiação (Rn)}

A Figura 7 estão apresentadas as informações do Rn, onde foi possível observar a variação do Rn de 400 a $700 \mathrm{~W} \mathrm{~m}^{-2}$ para as datas proposta do estudo. Diante disso, cabe ressaltar que a tonalidade lilás clara corresponde ao intervalo do $\mathrm{Rn}, 410$ a $555 \mathrm{~W} \mathrm{~m}^{-2}$, localizando em áreas com os solos expostos e urbanizadas, com pouca ou sem nenhuma cobertura vegetal.

As áreas com pouca ou sem nenhuma vegetação apresentaram valores elevados de albedo $(\alpha) \mathrm{e}$ baixo valores de saldo de $\mathrm{Rn}$ como no caso dos mapas de albedo do dia 30/01/2019 e 27/09/2019, e nos mapas do Rn para as respectivas datas, ou seja, nas áreas onde o $\alpha$ é alto o Rn é baixo. Cabe ressaltar, que esta relação se deve ao fato da combinação desses dois parâmetros físicos onde o $\alpha$ influencia no balanço de ondas curtas e o Rn no balanço de ondas longas.

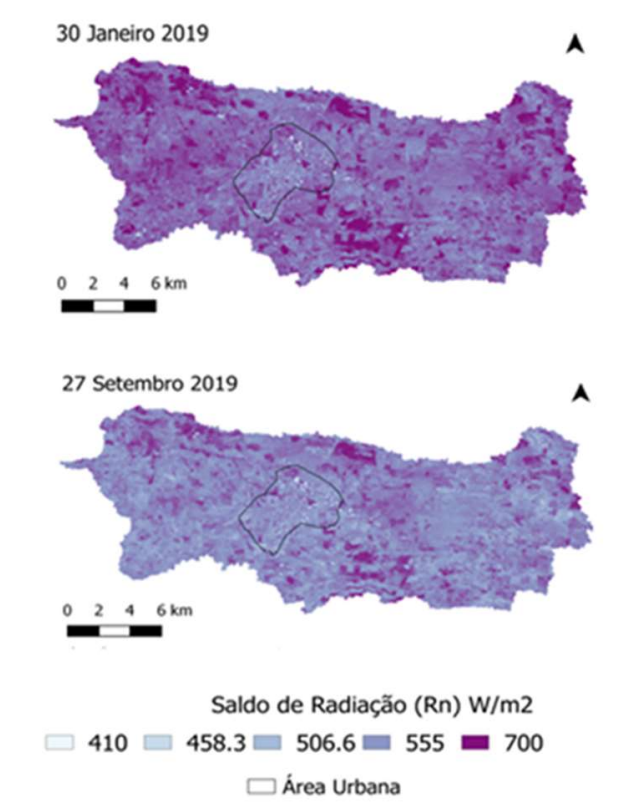

Figura 7: Mapas do saldo de radiação (Rn).

\section{Fluxo de Calor no Solo (G)}

A Figura 8 ilustra os mapas de fluxo de calor no solo (G) que representam a parcela do Rn que chegou na superfície da área de estudo.

Comparando-se as Figuras 8 e 3, verifica-se que as áreas com os pixels vigorados do NDVI, o G é inferior a $145 \mathrm{~W} \mathrm{~m}^{-2}$. Isso em virtude do mês de janeiro que está inserido no período chuvoso e consequentemente a vegetação apresenta uma densidade foliar mais elevada, impedido que a fração de Rn chegue ao solo. Além disso, o comportamento contrário ocorre para a data 27/09/2019 por causa do período de estiagem (período sem chuvas), e como resultado há a perda da densidade foliar da vegetação. 


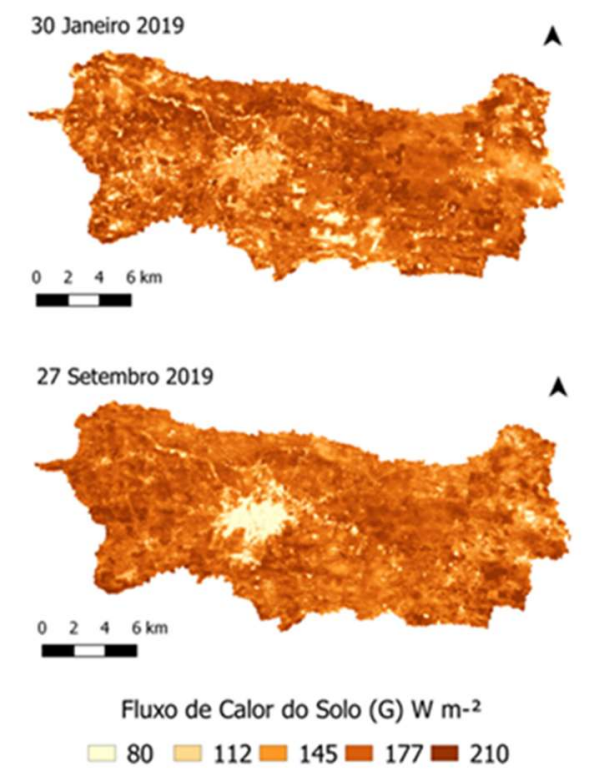

Figura 8: Mapas do fluxo de calor no solo $(G)$.

\section{Fluxo de Calor Sensível (H)}

Para a estimativa do $\mathrm{H}$, o algoritmo SEBAL necessita de processos interativos e neste trabalho foi preciso 6 interações para atingir o Erro Relativo menor que 1 \%. Para a calibração desses processos deu-se a partir da escolha dos pixels quentes e frios.

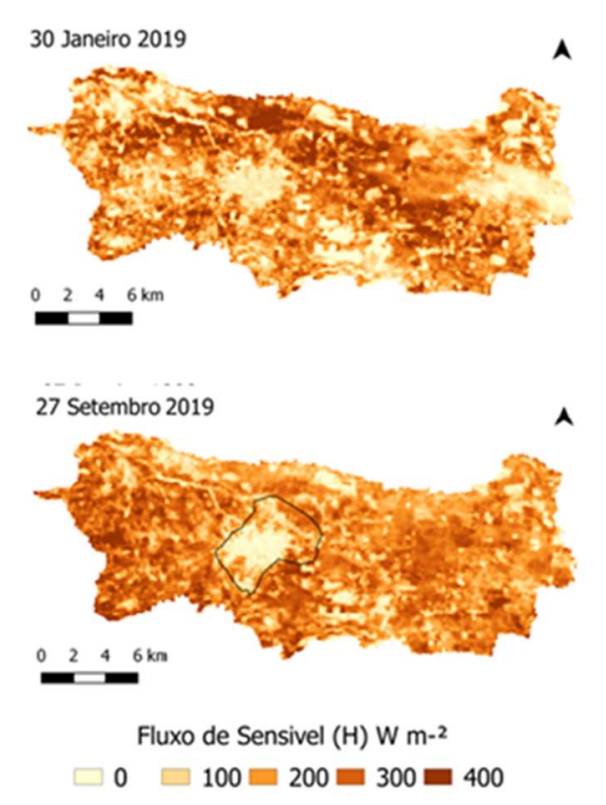

Figura 9: Mapa do Fluxo de Calor Sensível (H).

A Figura 9 ilustra os $\mathrm{H}$ para as datas estudas, apresentando valores variando de 0 à de $400 \mathrm{~W} \mathrm{~m}^{-2}$, onde observa-se que os maiores valores de $\mathrm{H}$ estão relacionados ao baixo índice vegetativo e as altas temperaturas, esta relação pode ser melhor observada quando comparados pixel a pixel dos mapas da Figura 6, 7 e 8. Portanto, quando ocorre essa relação, a maior fração de energia líquida disponível é direcionada para o aquecimento do ar e do solo, e por isso ocorre uma redução da energia destinada para a ET em forma de calor latente, tornando os componentes H e LET inversamente proporcionais. 


\section{Fluxo de Calor Latente (LET)}

Na Figura 10, estão apresentados os valores de LET variando de 0 a $600 \mathrm{~W} \mathrm{~m}^{-2}$ durante o período das cenas de estudo. Além disso, observa-se que os maiores valores foram para a data 30/01/2019, pelo fato que essas áreas apresentavam vegetação densa e os solos encontravam-se encharcados com água. Por outro lado, os menores valores de LET foram para os locais com o solo exposto.
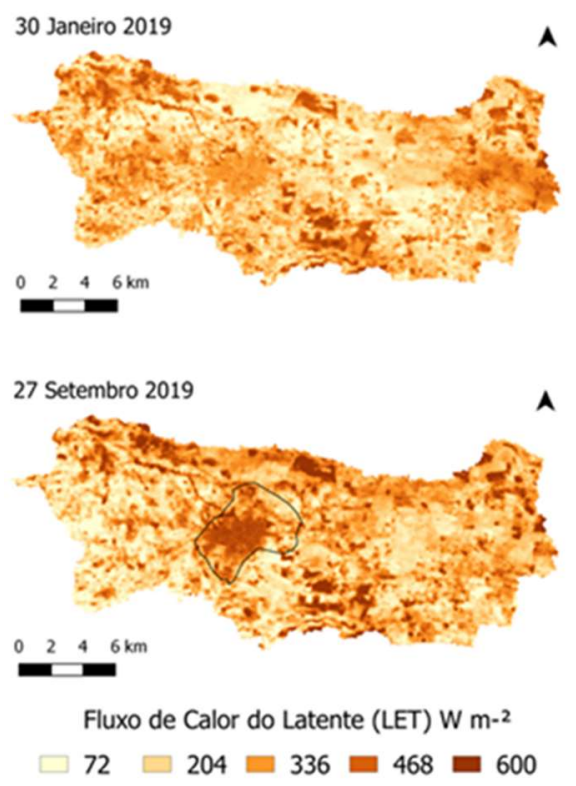

Figura 10: Mapa de calor latente.

\section{Evapotranspiração de Real (ETR)}

Na Figura 11, estão apresentados os valores de Evapotranspiração Real (ETr) variando de 0 a $17 \mathrm{~mm}$ $\mathrm{dia}^{-1}$, onde constata-se que a tonalidade vermelha corresponde as áreas de solo exposto e vegetação rala.

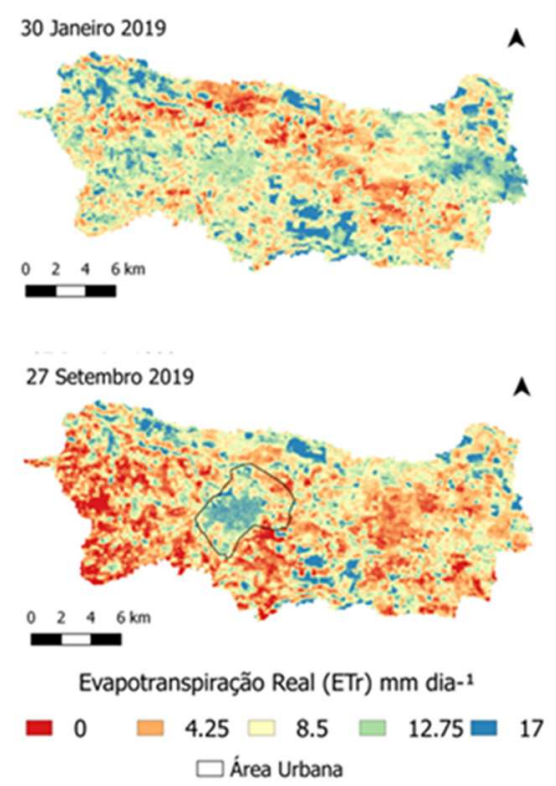

Figura 11: Mapa de Evapotranspiração Real (ETr).

Por meio da Figura 11, observa-se que a ET média diária para o mês de janeiro foi de 9,91 mm dia ${ }^{-1}$, sendo que esse mês está inserido no período chuvoso da região e apresenta precipitação acumulada. Diante 
disso, cabe ressaltar que o aumento da ETr nesse mês é decorrente da maior disponibilidade de água no solo que fica vulnerável aos efeitos das radiações solares, assim, consequentemente, intensificando o processo de ETr. Por outro lado, para a cena inserida no mês de setembro, ocorre condições meteorológicas distintas, onde a umidade, velocidade do vento e precipitação são menores.

\section{Análise Estatística}

Na Tabela 2 estão apresentados os valore de ETO e ETr para a área de estudo.

Tabela 2: Análise estatísticas de ETO e ETr.

\begin{tabular}{lllll}
\hline Data das Imagens & (ET0) & (ETr) & EA (mm/dia) & ER (\%) \\
\hline $30 / 01 / 2019$ & 5,15 & 9,91 & 4,76 & 48,03 \\
$27 / 09 / 2019$ & 2,75 & 2,67 & 0,08 & 3,00 \\
\hline
\end{tabular}

Legenda: (ETO) Evapotranspiração de referência; (ETr) Evapotranspiração calculada pelo SEBAl; (EA) Erro Absoluto; (ER) Erro Relativo.

Por meio da Tabela 2, observa-se que os maiores erros tanto absoluto quanto relativo foram constatados para a data de 30/01/2019, tendo e EA de 4,76 e ER de $48,03 \%$, respectivamente. Por outro lado, observa-se que os resultados para a data 27/09/2019 (período de estiagem) são bem satisfatórios tendo erros relativos e absolutos baixos, apresentando valores de EA e ER de $0,08 \mathrm{~mm} \mathrm{dia}^{-1}$ e $3 \%$, respectivamente.

Comparando os resultados dos erros EA e ER com outros autores, verifica-se que os resultados para a data no mês de janeiro foram insatisfatórios. Diante disso, Machado et al. (2014) encontraram EA de 0,32 mm dia ${ }^{-1}$ e ER 7,17\% para o litoral sul do Estado do Pernambuco no dia 28 de janeiro. Já no dia 02 de janeiro de 2012, Coelho (2016) constatou EA de 2,2 $\mathrm{mm} \mathrm{dia}^{-1}$. Além disso, cabe ressaltar que um autor encontrou diferença de 0 a 2,7 mm durante o período de julho a outubro em áreas irrigadas nos cultivos de trigo no município de Piraí do Sul no Estado do Paraná.

Nesta pesquisa, o mês de setembro apresentou os menores erros absolutos entre as estimativas realizadas através do algoritmo SEBAL, isso é decorrente pelo fato que esse algoritmo tenha sido desenvolvido e calibrado para regiões de clima árido e semiárido, uma vez que o período que foi realizado o estudo é caracterizado pela baixa pluviosidade, apresentando Ts elevadas e umidade relativa baixa.

Da mesma forma, as datas que não houveram nenhuma precipitação acumulada apresentaram-se os menores valores para os erros absolutos (Tabela 1). Por outro lado, esse mesmo fator causou a superestimativa da ETr para o mês de janeiro, decorrente das condições metrológicas distinta em relação das circunstanciais validadas para o SEBAL.

O método desenvolvido por Allen et al. (2002) apresentou algumas limitações, a começar pela subjetividade na escolha dos pixels âncoras, quentes e frios, podendo influenciar negativamente na estimativa do $\mathrm{H}$, apesar deste estudo seguir uma metodologia para a escolha, a seleção dos pixels é de forma empírica, ou seja, de forma manual. Além disso, existe também limitações para as regiões com declividades acima de suave ondulado, tendo em vista que o algoritmo exige que a superfície do terreno seja plana.

O método de Penman-Monteith-FAO usado para o cálculo de ETO, necessitando de dados meteorológicos obtidos em estações meteorológicas e que podem apresentar defeitos nos aparelhos de 
medições. Tal fato influencia na calibração do algoritmo conforme a metodologia adotada neste trabalho, uma vez que a estimativa de ETr é baseada na de ETO. Apesar disso, O método de Penman-Monteith-FAO faz referência a ET de uma extensa superfície de grama verde e altura uniforme regada e com crescimento constante, situação distinta da região da área em estudo.

\section{CONCLUSÕES}

O algoritmo SEBAL mostrou-se muito eficiente para realizar estimativas dos componentes do balanço de energia utilizando imagens do satélite LANDSAT-8 (OLI). Além disso, constatou-se uma forte influência da vegetação e da precipitação acumulada para as datas em estudo, onde verificou-se média de $9,91 \mathrm{~mm} \mathrm{dia}^{-1}$ para a data de 30 de janeiro de 2019. Por outro lado, as menores taxas de ETr correspondem as áreas que são desenvolvidas atividades antrópicas com potencial impactante para o meio ambiente, apresentando o solo exposto sem nenhuma cobertura vegetal.

O SEBAL apresentou valores estimados para o período de estiagem coerente, porém para a data de alta pluviometria os valores dos erros mostraram-se muito elevados, evidenciando que esse método precisa de ajustes e calibração para períodos distintos (chuvoso e seco).

Por fim, espera-se que os resultados alcançados por meio deste estudo sirvam de base para a gestão sustentável dos recursos hídricos das bacias hidrográficas, principalmente as inseridas na região do Nordeste brasileiro.

\section{REFERÊNCIAS}

ALLEN, R. G.; TASUMI, M.; TREZZA, R.. Surface Energy Balance Algorithms for Land: Advance Training and Users Manual: Idaho Implementation. 2002.

ALLEN, R. G.; PEREIRA, L. S.; RAES, D.; SMITH, M.. Crop evapotranspiration: guidelines for computing crop water requirements - FAO Irrigation and Drainage paper 56. Rome, 1998.

BASTIAANSSEN, W. G. M.. Regionalization of surface flux densities and moisture indicators in composite terrain: $\mathbf{A}$ remote sensing approach under clear skies in Mediterranean climate. PhD (Dissertation) - CIP Data Koninklijke Biblioteheek, Den Haag, 1995a.

BASTIAANSSEN, W. G. M.. Regionalization of surface flux densities and moisture indicators in composite terrain: $A$ remote sensing approach under clear skies in Mediterranean climate. Dissertação (Mestrado) - CIP Data Koninklijke Biblioteheek, Den Haag, 1995b.

BASTIAANSEN, W. G. M.. SEBAL - based sensible and latent heat luxes in the irrigated Gediz Basin, Turkey. Journal of Hydrology, v.229, p.87-100, 2000.

BASTIAANSSEN, W. G. M.; MENENTI, M.; FEDDES, R. A.; HOLTSLAG, A. A. M.. The surface energy balance algorithm for land (SEBAL). Part 1, formulation. Journal of Hydrology, v.212-213, p.198-212, 1998.
BEZERRA, B. D.; SILVA, B. D.; FERREIRA, N. J.. Estimativa da evapotranspiração real diária utilizando-se imagens digitais TM-Landsat 5. Revista brasileira de Meteorologia, v.23, n.3, p.305-317, 2008

BRUTSAERT, W.. Evaporation into the atmosphere: Theory history, and applications. Boston, 1982.

COELHO, V. H. R.. Estimativa da recarga subterrânea em bacia hidrográfica do semiárido pernambucano a partir de técnicas de sensoriamento remoto e sistemas de informações geográficas. Tese (Doutorado em engenharia civil) - Universidade Federal de Pernambuco, Recife, 2016.

GIACOMONI, M. H.. Estimativa da evapotranspiração regional por meio de técnicas de sensoriamento remoto integradas a modelos de balanço de energia: aplicação no estado do Rio Grande do Sul. Dissertação (Mestrado em recursos hídricos e saneamento ambiental) - Universidade Federal do Rio Grande do Sul, Porto Alegre, 2005.

GONÇALVES, T. S.; LEAL, L. R. B.. Potencialidades hídricas no Aquífero Carstico Salitre na região de Irecê, Bahia. Águas Subterrâneas, v.32, n.2, p.191-199, 2018.

MACHADO, C. C.; SILVA, B. B. D.; ALBUQUERQUE, M. B.; GALVÍNCIO, J. D.. Estimativa do balanço de energia utilizando imagens TM-Landsat 5 e o algoritmo SEBAL no litoral sul de Pernambuco. Revista Brasileira de Meteorologia, v.29, n.1, p.55-67, 2014. 
MENEZES, S. J. M. D. C. D.; SEDIYAMA, G. C.; SOARES, V. P.; GLERIANI, J. M.; ANDRADE, R. G.. Estimativa dos componentes do balanço de energia e da evapotranspiração em plantios de eucalipto utilizando o algoritmo SEBAL e imagem LANDSAT 5-TM. Revista Árvore, v.35, n.3, p.649657, 2011.

MORSE, A.; ALLEN, R. G.; TASUMI, M.; KRAMBER, W. J.; TREZZA, R.; WRIGHT, J.. Application of the SEBAL Methodology for Estimating Evapotranspiration and Consumptive Use of Water Through Remote Sensing. Final Report, 2001.

OLIVEIRA, L. M. M. D.. Estimativa da evapotranspiração real por sensoriamento remoto na bacia do rio Tapacurá-PE.
2012.

PAULSON, C. A.. The mathematical representation of wind speed and temperature profiles in the unstable atmospheric surface layer. Journal of Applied Meteorology, v.9, n.6, p.857-861, 1970.

SILVA, B. B.; BEZERRA, M. V. C.. Determinação dos fluxos de calor sensível e latente na superfície utilizando imagens TMLandsat 5. Revista brasileira de agrometeorologia, v.14, n.2, p.174-186, 2006.

SILVA, B. B. D.; MERCANTE, E.; BOAS, M. A. V.; WRUBLACK, S. C.; OLDONI, L. V.. Satellite-based ET estimation using Landsat 8 images and SEBAL model. Revista Ciência Agronômica, v.49, n.2, p.221-227, 2018.

A CBPC - Companhia Brasileira de Produção Científica (CNPJ: 11.221.422/0001-03) detém os direitos materiais desta publicação. Os direitos referem-se à publicação do trabalho em qualquer parte do mundo, incluindo os direitos às renovações, expansões e disseminações da contribuição, bem como outros direitos subsidiários. Todos os trabalhos publicados eletronicamente poderão posteriormente ser publicados em coletâneas impressas sob coordenação da Sustenere Publishing, da Companhia Brasileira de Produção Científica e seus parceiros autorizados. Os (as) autores (as) preservam os direitos autorais, mas não têm permissão para a publicação da contribuição em outro meio, impresso ou digital, em português ou em tradução. 